\title{
Molecular biomarkers and histological parameters impact on survival and response to first- line systemic therapy of metastatic colorectal cancer patients
}

\author{
Martina Rebersek, Tanja Mesti, Marko Boc, Janja Ocvirk \\ Department of Medical Oncology, Institute of Oncology Ljubljana, Ljubljana, Slovenia
}

Radiol Oncol 2019; 53(1): 85-95.

Received 30 August 2018

Accepted 20 September 2018

Correspondence to: Assoc. Prof. Janja Ocvirk, M.D., Ph.D., Department of Medical Oncology, Institute of Oncology Ljubljana, Zaloska 2, SI1000 Ljubljana, Slovenia. Phone: +386 15879 285: E-mail: jocvirk@onko-i.si

Disclosure: No potential conflicts of interest were disclosed.

Background. Histological parameters of primary tumour and nodal metastases are prognostic factors for survival of operable colorectal (CRC) patients, but not predictive for response rate of systemic therapy. KRAS mutations in codons 12 and 13 were first recognized as a predictive factor for resistance to anti-EGFR monoclonal antibodies. Not all patients with wild-type KRAS (wTKRAS) respond to anti-EGFR antibody treatment. Additional mechanisms of resistance may activate mutations of the other main EGFR effectors pathway, such as other mutations in RAS gene, mutations in P13K and PTEN expression.

Patients and methods. In the prospective study prognostic and predictive impact of histological parameters of primary tumour, KRAS and BRAF mutations on overall survival (OS) and objective response (OR) rate of metastatic CRC $(\mathrm{mCRC})$ patients treated with $1^{\text {st }}$ line systemic therapy were analysed. We additionally retrospectively analysed other mutations in RAS genes and their impact on survival and time to progression.

Results. From November 2010 to December 2012, we enrolled 154 patients in the study, 95 men and 59 women. Mutations in KRAS gene and V600E BRAF gene were found in $42 \%$ and in $3 \%$ of patients, respectively. Median OS of the patients with T1, T2 and T3 tumour was 65.4 months $(95 \% \mathrm{Cl}, 55.7-75.6)$ while in patients with T4 tumour, lymphangiosis, vascular and perineural invasion it has not been reached yet. Median OS of the patients with G1, G2 and G3 of tumour differentiation was $65.6(95 \% \mathrm{Cl}, 53.7-77.5)$ and 25.3 months $(95 \% \mathrm{Cl}, 16.6-34.1)$, respectively. Median OS of the patients with stage NO, N1 and N2 was 65.6 (95\% Cl, 56.4-74.8) and 58.0 months (95\% Cl, 21.9-94.2), respectively. Median OS of WTKRAS and mutated KRAS patients was 56.5 (95\% Cl, 48.2-64.9) and 58 months (95\% Cl, 52.6-63.4), respectively. Median OS of mutated codon 12 and codon 13 patients was 57 (95\% Cl, 50.9-64.4) and 44 months (95\% $\mathrm{Cl}, 40.1-48.4)$, respectively. Median OS of WTBRAF and of mutated BRAF patients was $59.2(95 \% \mathrm{Cl}, 52.5-65.9)$ and 27.6 months $(95 \% \mathrm{Cl}, 12.6-42.5)$, respectively. wTKRAS significantly affected the response to the first systemic therapy ( $\mathrm{p}=$ 0.028 ), while other parameters did not affected it, $p=0.07$. In 14 patients (17\%), additional mutations in NRAS gene, codon 61 and codon 146 were found. Median OS of wtNRAS, codon 61 and 146 patients was 67.1 months (50.3-67.6) while median OS of mutated NRAS patients has not been reached yet ( $p=0.072$ ). Median time to progression of WTNRAS, codon 61 and 146 patients was 11.7 months (10.4-14.5) while median time to progression of mutated NRAS patients was 7.9 months (6.1-11.0), ( $p=0.025)$.

Conclusions. Mutated BRAF, N2 and G3 of primary tumour were poor prognostic factors for OS in mCRC patients. WTKRAS significantly affected the response to the first line systemic therapy. Histological parameters included in the analysis and mutated BRAF did not affect significantly the efficacy of $1^{\text {st }}$ line systemic therapy in $\mathrm{mCRC}$ patients.

Key words: metastatic colorectal cancer; chemotherapy; targeted therapy; histological parameters; biomarkers 


\section{Introduction}

Colorectal cancer (CRC) is one of the most common cancer and one of the leading causes of cancer death in the world. It is one of the most common cancers in Slovenia and, according to the Cancer Registry of Slovenia, 1353 new patients were diagnosed with CRC in 2015. ${ }^{1}$ Metastatic disease is still incurable, with $5 \%$ five-year overall survival (OS) without treatment. With the introduction of new chemotherapy, using oxaliplatin and irinotecan in the current management of metastatic disease, in combination with biologicals, targeting epidermal growth factor- mediated growth regulatory pathway and the vascular endothelial growth factormediated angiogenesis pathway we can prolong the progression-free survival (PFS) and OS of these patients. ${ }^{2-10}$ In selected patients with appropriate combination of therapy and surgery we can achieve approximately a 50\% five-year OS.

The development of CRC is a multistep process, which accumulates different gene mutations, chromosomal abnormalities and epigenetic changes. ${ }^{11}$ The mutations within KRAS proto-oncogene, predominately within codons 12 and 13, activate RAS/ RAF signalling and are thought to occur early in carcinogenesis of CRC. The KRAS status is the first molecular marker to predict the response to anti-EGFR monoclonal antibodies cetuximab and panitumumab in metastatic CRC (mCRC) patients, and it needs to be determined before deciding in favour of treatment with anti-EGFR antibodies. As the KRAS mutations occur early in CRC formation, there is a high concordance between the $K R A S$ mutations of primary tumour and metastases, which was confirmed in previous studies. ${ }^{11}$ In the retrospective study, de Roock et al. raised the possibility that the patients with the KRAS mutation in codon 13 might have benefited from antiEGFR antibodies treatment. ${ }^{12,13}$ The mutations in KRAS gene were found in approximately 30 to $40 \%$ of $\mathrm{mCRC}$ patients, reported in previous literature, but, only 40 to $60 \%$ of these patients with wtKRAS will respond to anti-EGFR antibodies treatment. Therefore, other molecular markers downstream of EGFR in the RAS/RAF/MAPK pathway and other effector pathways were found to be involved to predict the response to specific systemic therapy.

The BRAF gene encodes a serine/threonine protein kinase of the RAS/RAF/MEK/ERK kinase pathway and it is also involved in CRC carcinogenesis. ${ }^{11,14,15}$ The most common mutation of the BRAF gene is V600E which is found in approximately 5 to $9 \%$ of $\mathrm{mCRC} .^{15,16}$ The same was reported in our previous study carried on Slovenian patients with CRC where the BRAF V600E mutation was found in $5.1 \%$ of patients. ${ }^{16}$ Previous retrospective studies suggested that mutated $B R A F$ was a marker of resistance to anti-EGFR therapy and that the patients with mutated $B R A F$ had significantly shorter PFS and OS than the patients with wtBRAF tumours. ${ }^{14,15}$ The mutations in the KRAS and BRAF genes have been reported to be mutually exclusive. In the retrospective analysis by Fariña- Sarasqueta et al., it was also shown that the BRAF V600E mutation was an independent prognostic factor for the OS of patients with CRC in stages II and III, while the KRAS mutations did not have any effect on the OS of these patients. ${ }^{17}$ They concluded that the prognostic role of the KRAS mutations in an adjuvant setting had to be determined. In published clinical studies the BRAF V600E mutation in MCLC is conferred to a poor prognosis regardless of treatment, but these patients may have some benefit from the treatment with cetuximab in combination with chemotherapy as the first-line therapy, except for the patients in whom the disease has progressed after the first-line therapy. ${ }^{15,17}$ The status of mutations in the RAS gene is a new molecular predictive factor for response to treatment with EGFR inhibitors in mCRC. These mutations in the RAS gene, in the codons 12, 13, 61 and 146, according to the literature data, are about $15 \%$, and they are determined from autumn 2013 at our Institute of Oncology.3,4,9

The aim of this prospective study was to analyse overall response rate (ORR), time to progression (TTP) and OS of the patients with MCRC treated with first-line systemic therapy in respect of histological parameters of primary tumour KRAS and $B R A F$ status. We additionally retrospectively analysed other mutations in RAS genes and their impact on OS and TTP.

\section{Patients and methods}

\section{Patients and treatment}

In the study, 154 patients with histologically confirmed mCRC, primarily metastatic or progressed during or after adjuvant therapy were prospectively analysed. They were treated according to the national, ESMO and NCCN guidelines, including performance status of patients and comorbidity. They were treated with chemotherapy, including fluoropirimidins, capecitabine or 5-fluorouracil (5FU), oxaliplatin or irinotecan in combination with biologicals, bevacizumab or cetuximab in respect of previously determined KRAS status. The treat- 
ment was continued according to the RECIST criteria, until the planned operation or until the progression of disease or toxicity occurred.

All relevant data from medical files were collected and entered into the data base. Baseline data was analysed with regard to age, sex, primary site (colon and rectum), number and location of metastases. Efficacy was evaluated according to the response evaluation criteria in solid tumours (RECIST, version 1.1) by using computed tomography (CT) scans, magnetic resonance scans, abdominal ultrasound, chest X-ray, bone scans, clinical examination and laboratory tests. ${ }^{17}$ Toxicity was assessed according to the National Cancer Institute common toxicity criteria for adverse events (NCICTCAE), version 4.03. The study was approved by the Institutional Review Board Committee and was carried out according to the Declaration of Helsinki.

\section{Hystology}

From the histological findings, data on the tumour size, the radical nature of the primary tumour operation, the presence of vascular and perinevral invasion, carcinogenic lymphangiosis, the degree of differentiation, the presence of outbreaks of malignant cells and the number and severity of regional lymph nodes were collected.

\section{Biomarker analysis}

DNA for molecular analysis was extracted from formalin-fixed, paraffin-embedded tumour tissue of primary tumours or metastases with at least $70 \%$ of tumour cells. TheraScreen KRAS Mutation $\mathrm{Kit}^{\circledR}$ (Roche Applied Science, Mannheim, D) was used to determine seven most common mutations in codons 12 and 13 of the KRAS gene. The V600E mutation in BRAF was detected by end-point genotyping using the TaqMan MGB probes (Applied Biosystems, Warrington, UK) as described previously. ${ }^{16}$ The mutation V600E in BRAF in positive tumour samples was confirmed by direct sequencing after amplification of the exon 15 of the BRAF gene. ${ }^{16}$ Retrospectively additional mutations in NRAS gene, in KRAS codon 61 and 146 were determined with the same method as mutations in KRAS codon 12 and codon 13.

\section{Statistical methods}

The primary end-points of the analysis were overall response rate (ORR), based on RECIST criteria,
OS and time to progression (TTP) according to the KRAS and BRAF status.

The $\chi^{2}$-test was used to compare ORR, OS and TTP between groups, with 95\% confidence intervals (CI) calculated for the medians. OS and TTP were estimated by using Kaplan-Meier Estimates and compared using the log-rank test. TTP was measured in all patients from the beginning of the first-line systemic chemotherapy to the first evidence of progression. The duration of OS was calculated from the beginning of systemic treatment until the date of death. P value $<0.05$ was considered statistically significant. Statistical data were obtained using the SPSS software package PASW statistics 18.0 .

The relationship between treatment efficacy and mutations in KRAS and BRAF gene and histological characteristics of the tumour (tumour size, presence of vascular and perineural invasion, lymphangiosis, degree of differentiation, number of affected regional lymph nodes) were evaluated by the method of the nominal logistic regression. With the Cox proportional hazards model, we evaluated the impact of histological factors, the number of affected nodes, and mutations in KRAS and $B R A F$ gene on OS or TTP. Survival was calculated by Kaplan-Meier method to compare groups of patients, and we used the log-rank test. We marked statistically significant differences at $p<0.05$. The same statistical methods were used for OS and TTP in the case of retrospectively determined additional mutations in RAS gene.

\section{Results}

\section{Patient's characteristics}

In total, 154 patients with mCRC who received first-line therapy between November 2010 and December 2012 were included in this prospective analysis. The cut-off date for the present analysis was April 2011. All patients were treated at the Institute of Oncology Ljubljana, all were Caucasian. The median age was 62 years (range 27-86) and the majority of the patients were males (61\%). Most of the patients had mCRC $(71.4 \%)$. One hundred and four patients had primary metastatic disease (59.1\%). The most common sites of metastases were liver and lung. The most common therapies the patients received were irinotecan, capecitabine with bevacizumab (29.5\%) and oxaliplatin, capecitabine with cetuximab (22.1\%). Twenty-four patients $(13.6 \%)$ were treated only with chemotherapy, capecitabine in monotherapy, or with fluoro- 
TABLE 1. Patient's baseline characteristics

\begin{tabular}{ll}
\hline Patient's characteristics & Number (\%) \\
\hline Medium age (years) & 62 \\
Gender & $59(38)$ \\
female & $95(62)$ \\
male & \\
WHO performance status & $36(23)$ \\
0 & $109(71)$ \\
1 & $9(6)$ \\
2 & $112(73)$ \\
Tumour location & $42(27)$ \\
colon & $89(58)$ \\
rectum & $68(44)$ \\
Primary metastatic & \\
Liver metastases &
\end{tabular}

TABLE 2. Disease characteristics

\begin{tabular}{ll}
\hline Disease characteristics & Number (\%) \\
\hline pT4 of primary tumour & $35(23)$ \\
Affected regional lymph nodes (N) & \\
N0 (no affected regional lymph nodes) & $34(22)$ \\
N1 (1 to 3 affected regional lymph nodes) & $61(40)$ \\
N2 (more than 3 affected regional lymph & $59(38)$ \\
nodes) & \\
Vascular invasion & $22(14)$ \\
Perineal invasion & $25(16)$ \\
Lymphangiosis & $27(17)$ \\
Grade of differentiation & \\
G1 (well) & $10(6)$ \\
G2 (medium) & $131(85)$ \\
G3 (poorly) & $13(8)$ \\
KRAS gen & $89(58)$ \\
non-mutated (wild-type) & $65(42)$ \\
mutated & \\
KRAS mutation & $48(73)$ \\
Codon 12 & $17(27)$ \\
codon 13 & $150(97)$ \\
BRAF gen & $4(3)$ \\
non-mutated (wild-type) & \\
mutated & \\
\hline
\end{tabular}

pirimidines in combination with oxaliplatin or irinotecan. Patients' baseline characteristics and are shown in Table 1, disease characteristics are shown in Table 2.

Median follow-up was 15 months. Of the enrolled patients, $71 \%$ had WHO performance status 1 , and $73 \%$ of them the CRC primary metastatic disease was confirmed in $58 \%$ of patients. Median time to first progression after primary treatment of operable disease was 20 months. Because of a small number of R1 resections of the primary tumour (in 4 patients), perforations of the primary tumour during surgery (in 5 patients) and extramural tumour deposits (in 4 patients), these three parameters were excluded from the analysis. In $42 \%$ of

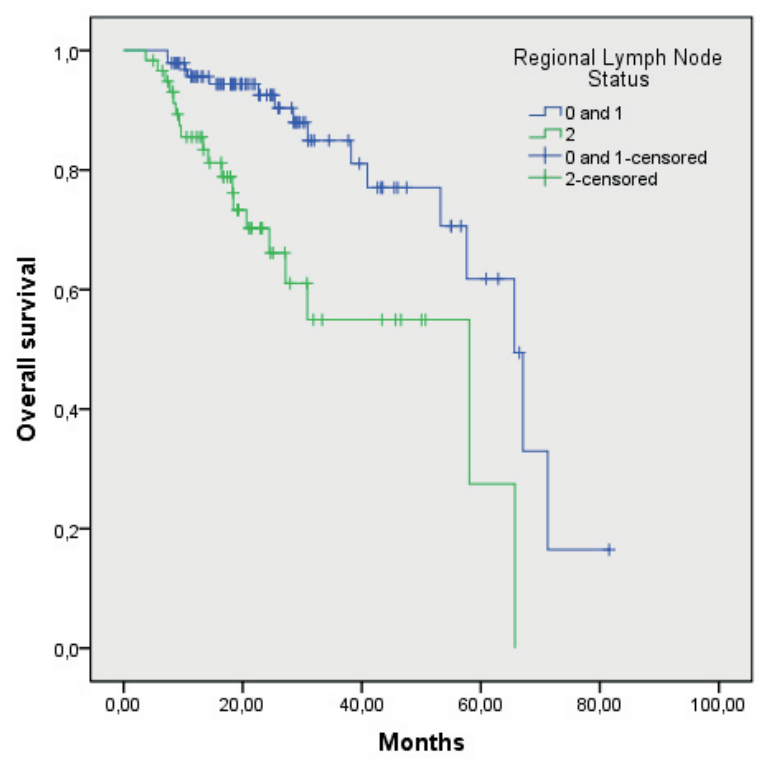

FIGURE 1. Overall survival of patients according to regional lymph node status $(p=0.000)$.

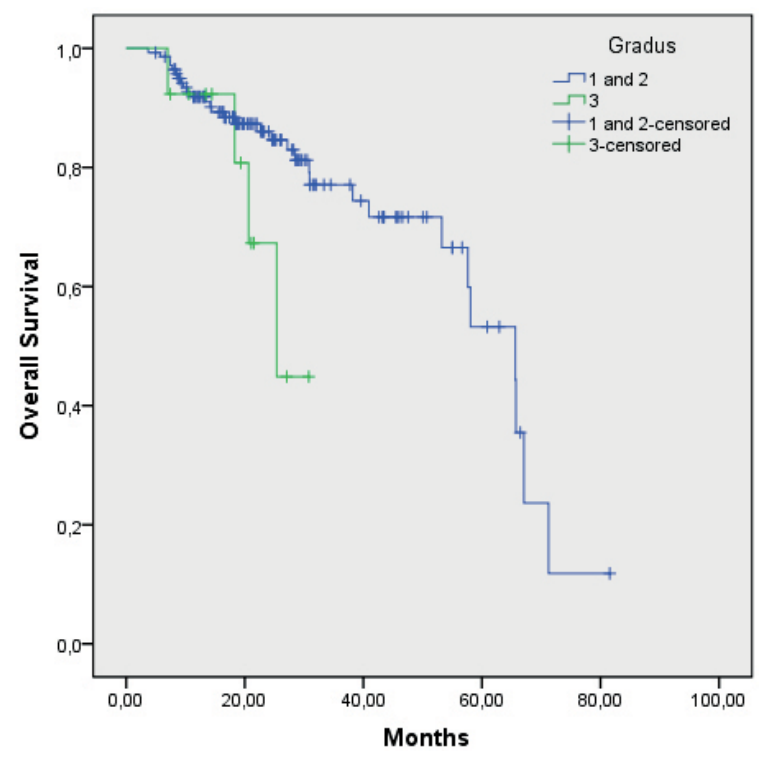

FIGURE 2. Overall survival of patients according to tumour grade of differention $G 1$ and $G 2$ versus $G 3$.

patients, the mutations in the KRAS gene were found, most frequently in codon $12(73 \%)$, whereas the V600E mutation in the BRAF gene was found in $3 \%$. The mutations of the KRAS or BRAF gene were detected in total in 76 patients $(43.4 \%)$.

Medium OS of the patients with T1, T2 and T3 primary tumour was 65.4 months (55.7-75.6), while in the patients with T4 primary tumour, it has not been reached yet $(p=0.08)$. Median OS of the patients without vascular invasion was 65.6 months 


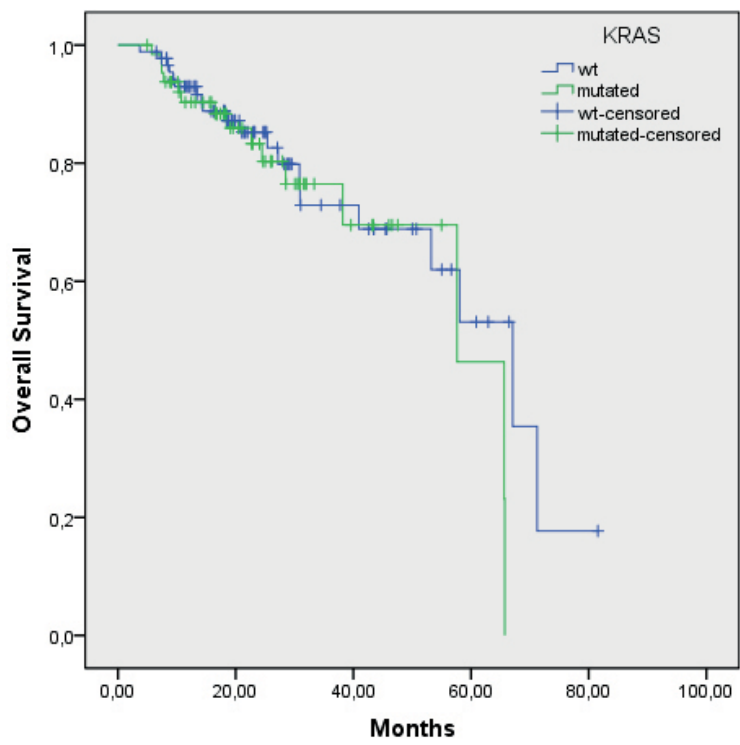

FIGURE 3. Overall survival of WtKRAS and mutated KRAS gene patients $(p=0.47)$.

(53.5-77.7), and in the patients with vascular invasion, it has not been reached yet. Median OS of the patients without perinevral invasion was 65.6 months (47.9-83.3), and in the patients with perinevral invasion, it has not been reached yet. Median OS of the patients without lymphangiosis was 65.6 months (53.5-77.6), and in the patients with lymphangiosis, it has not been reached yet. Median OS of the patients with grade 1 and 2 of tumour differentiation was 65.6 months (53.7-77.5), and of the patients with grade 3 of tumour differentiation, it was 25.3 months $(16.6-34.1)(p=0069)$. Median OS of the patients without affected regional lymph nodes or less than 3 of them (N0 and N1) was 65.6 months (56.4-74.8), and of the patients with more than 3 affected regional lymph nodes (N2), it was 58.0 months (21.9-94.2); the difference was statistically significant $(\mathrm{p}=0.000)$. OS of the patients according to regional lymph nodes and according to the grade of tumour differentiation are shown in Figure 1 and 2, respectively.

Median time to first progression after primary treatment of operable disease in the patients with the wtKRAS gene was 20 months (14.7-26.1), and in the patients with the mutated KRAS gene, it was 21 months (17.1-25.4) $(p=0.88)$. In the patients with the mutations in codon 12, it was 21 months (14.3-28.3), and in the patients with the mutations in codon 13, it was 16 months (11.5-20.7) $(\mathrm{p}=0.53)$. Median time to first progression after primary treatment of operable disease in the patients with the wtBRAF gene was 20 months (16.7-24.1), and

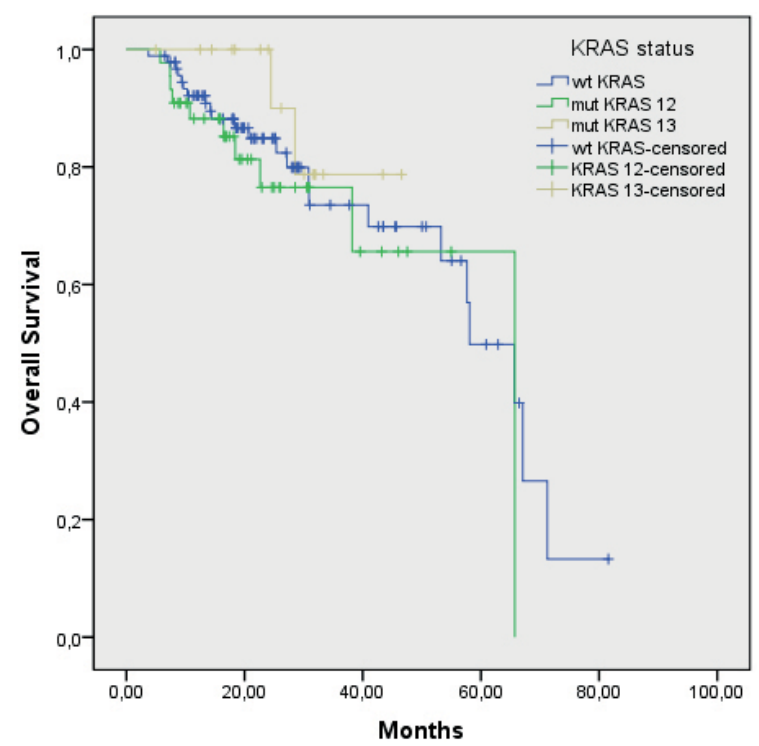

FIGURE 4. Overall survival of mutated KRAS patients in codon 12 and $13(p=0.40)$.

in the patients with the mutated $B R A F$ gene, it was 12 months $(1.8-23.1)(\mathrm{p}=0.14)$.

Median OS of the patients with the wtKRAS gene was 56.5 months (48.2-64.9), and in the patients with the mutated KRAS gene, it was 58 months (52.6-63.4 months; $p=0.47$ ) (Figure 3). In the patients with the mutation in codon 12, the median OS was 57 months (50.9-64.4), and in the patients with the mutation in codon 13 , it was 44 months (40.1-48.4 months; $\mathrm{p}=0.40$ ) (Figure 4). Median OS of the patients without mutation in the $B R A F$ gene was 59.2 months (52.5-65.9), and in the patients with the mutated BRAF gene, it was 27.6 months (12.6-42.5), the difference was statistically significant $(p=0.05)$ (Figure 5). The wtKRAS gene statistically significantly affected the response to the systemic therapy $(\mathrm{p}=0.028)$. Histological parameters included in the analysis and the BRAF gene mutation did not affect significantly the efficiency of first line systemic treatment.

Of 154 patients, 85 patients were wtKRAS and wtBRAF avaible for additional analysis, for 3 patients there was not possible to determine additional biomarker analysis from tumour tissue.

In 14 patients $(17 \%)$ additional mutations in RAS gene were determined. Two patients were NRAS mutated, in 8 patients KRAS codon 61 mutation was determined, 4 patients were KRAS codon 146 mutated.

Median OS of $68 \mathrm{wt} R A S$ patients was 27 months, median OS of 14 mutated RAS patients was 15 months. Median OS of wtNRAS, codon 61 and 


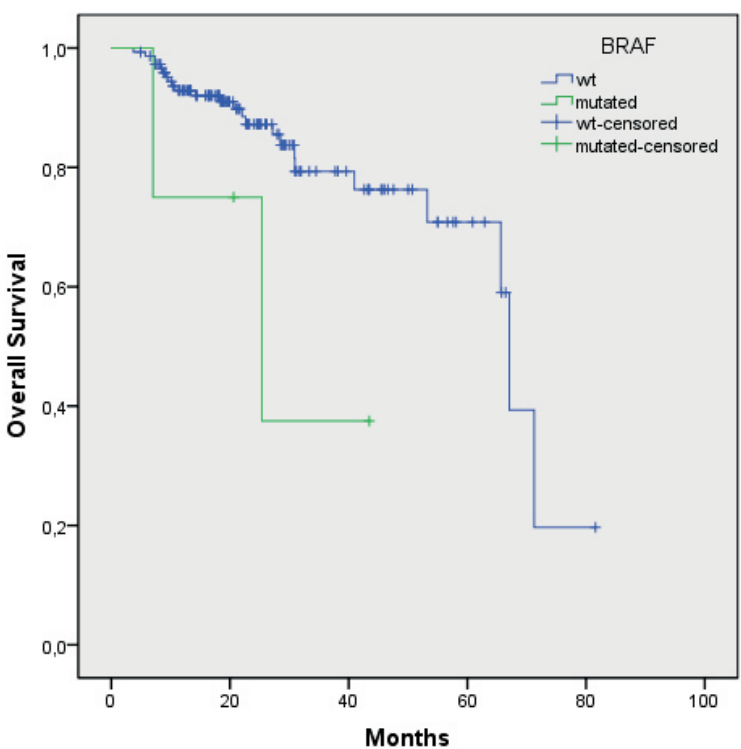

FIGURE 5. Overall survival of WtBRAF and mutated BRAF gene patients $(p=0.05)$.

TABLE 3. Response rate according to systemic treatment

\begin{tabular}{lll}
\hline Response rate & $\begin{array}{l}\text { Systemic chemotherapy+ } \\
\text { cetuksimab, number (\%) }\end{array}$ & $\begin{array}{l}\text { Systemic chemotherapy+ } \\
\text { bevacizumab, number (\%) }\end{array}$ \\
\hline Complete response & $7(13)$ & $6(17)$ \\
Partial response & $18(34)$ & $8(23)$ \\
Stable disease & $21(40)$ & $19(54)$ \\
Progression of disease & $7(13)$ & $2(6)$ \\
\hline
\end{tabular}

TABLE 4. Response rate according to mutations in codon 12 and 13

\begin{tabular}{lll}
\hline Response rate & $\begin{array}{l}\text { Codon } 12 \text { mutations, } \\
\text { number }(\%)\end{array}$ & $\begin{array}{l}\text { Codon } 13 \text { mutations, } \\
\text { number }(\%)\end{array}$ \\
\hline Complete response & $6(12)$ & $2(12)$ \\
Partial response & $5(11)$ & $6(35)$ \\
Stable disease & $23(49)$ & $7(41)$ \\
Progression of disease & $13(28)$ & $2(12)$ \\
\hline
\end{tabular}

146 patients was 67.1 months (50.3-67.6), in mutated NRAS, codon 61 and 146 patients, it has not been reached yet $(p=0.072)$ (Figure 6). Median TTP of wtNRAS, codon 61 and 146 patients, was 11.7 months (10.4-14.5), of mtNRAS, codon 61 and 146 patients, was 7.9 months $(6.1-11.0$ months; $\mathrm{p}=$ 0.025) (Figure 7).

\section{Efficacy}

In the patients with the wtKRAS gene, the objective response was achieved in $47 \%$ of the patients treated with chemotherapy and cetuximab and in $40 \%$

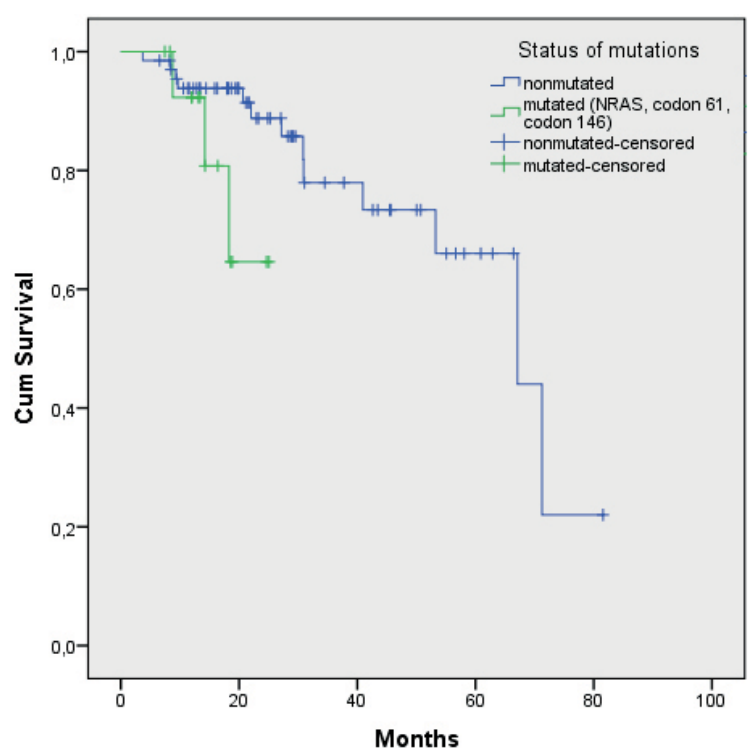

FIGURE 6. Median overall survival of WTNRAS, codon 61 and 146 patients and mutated NRAS, codon 61 and 146 patients $(p=0.072)$.

of the patients treated with chemotherapy and bevacizumab. Stable disease was achieved in $40 \%$ of the patients treated with chemotherapy and cetuximab and in $54 \%$ of the patients treated with chemotherapy and bevacizumab. The disease progressed in $13 \%$ of the patients who received chemotherapy and cetuximab, and in $6 \%$ of the patients who were treated with chemotherapy and bevacizumab.

In the patients with the mutated KRAS gene, the objective response was achieved in $23 \%$ of the patients with the mutation in codon 12 , and in $47 \%$ of the patients with the mutation in codon 13. Stable disease was achieved in $49 \%$ of the patients with the mutation in codon 12 and in $41 \%$ of the patients with the mutation in codon 13.The disease progressed during first-line treatment in $28 \%$ of the patients with the mutation in codon 12 and in $12 \%$ of the patients with the mutation in codon 13. Response rate according to systemic treatment and according to mutations in codon 12 and 13 are shown in Table 3 and 4, respectively.

\section{Toxicity}

The most common side effects according to the criteria of the National American Institute for Cancer - National Cancer Institute - common toxicity criteria (NCI - CTC, version 4.03) were of Grade 1 or 2. The adverse effects of Grade 3 were rare: diarrhea $(2 \%)$, allergic reaction to cetuximab $(2 \%)$, rash $(1 \%)$ and thromboembolic $(1 \%)$. Grade 4 adverse reactions were diarrhea $(1 \%)$, leukopenia $(1 \%)$, neu- 


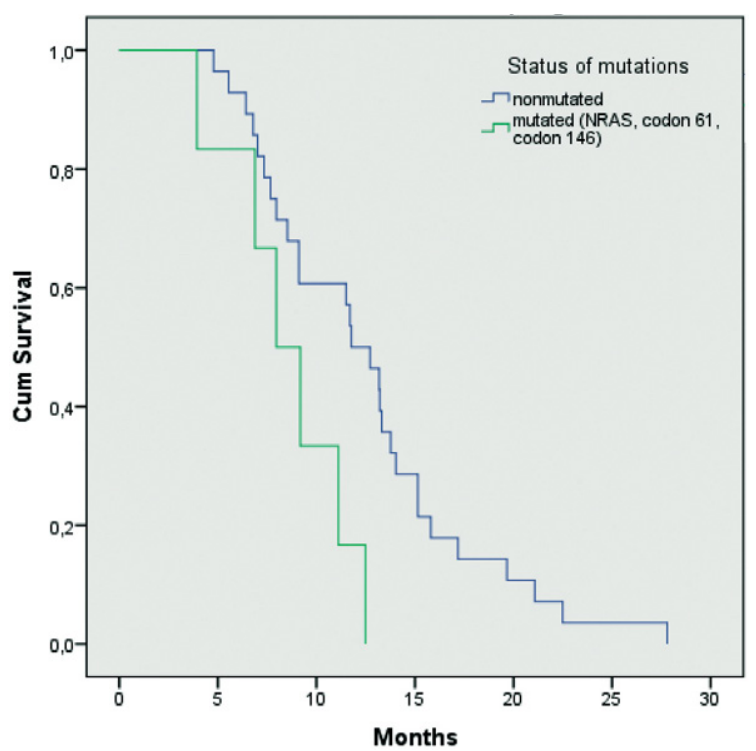

FIGURE 7. Median time to progression of WTNRAS, codon 61 and 146 patients, and mutated NRAS, codon 61 and 146 patients $(p=0.025)$.

tropenia (1\%) and febrile neutropenia (1\%). None of the patients died of adverse effects of systemic treatment (Table 5).

\section{Discussion}

To our knowledge a prospective clinical study in patients with CRC determining the prognostic and predictive significance of the histological characteristics of primary tumours with mutations in the KRAS and BRAF gene regarding response to treatment has not yet been published. Our study was the first of that kind. The study was conducted from November 2010 to December 2012 and154 patients were included, which was in line with the research plan. The percentage of patients with $\mathrm{T} 4$ tumour, G3 tumour, with more than 3 affected regional lymph nodes (N2), vascular, perinevral invasion and lymphangiosis were comparable with previously published data. ${ }^{2,18}$ Parameters as perforation of the primary tumour during surgery, presence of tumour deposits in subserosa, mesentery, peritonealised pericolic or perirectal mesenteric tissues and iresectability of the primary tumour, were excluded, because of the insufficient number

According to our results, poorly differentiated tumour cells and regionally advanced disease with a greater number of affected regional lymph nodes indicate a worse prognosis and a shorter survival of these patients. Other histological parameters of the primary tumour, such as the size of $\mathrm{T} 4$ tumour,
TABLE 5. Adverse effects of systemic treatment

\begin{tabular}{lllll}
\hline & $\begin{array}{l}\text { Grade } \mathbf{1} \\
\mathbf{n}(\%)\end{array}$ & $\begin{array}{l}\text { Grade 2 } \\
\mathbf{n}(\%)\end{array}$ & $\begin{array}{l}\text { Grade 3 } \\
\mathbf{n}(\%)\end{array}$ & $\begin{array}{l}\text { Grade 4 } \\
\mathbf{n}(\%)\end{array}$ \\
\hline $\begin{array}{lllll}\text { Haematological } \\
\text { leukopenia }\end{array}$ & $42(27)$ & $8(5)$ & 0 & $1(1)$ \\
neutropenia & $42(27)$ & $8(5)$ & 0 & $1+1$ \\
thrombocytopenia & $33(21)$ & $11(7)$ & 0 & $(1+1)^{*}$ \\
anaemia & $88(57)$ & $10(6)$ & 0 & 0 \\
Non-haematological & & & & 0 \\
alopecia & & & & \\
fatigue & $62(40)$ & $21(14)$ & $/$ & $/$ \\
nausea & $93(60)$ & $11(7)$ & 0 & $/$ \\
vomiting & $40(26)$ & $10(6)$ & 0 & $/$ \\
diarrhea & $14(9)$ & $9(6)$ & 0 & 0 \\
stomatitis & $23(15)$ & $29(19)$ & $2(2)$ & $1(1)$ \\
hand-foot syndrome & $4(3)$ & $2(2)$ & 0 & 0 \\
peripheral sensory neuropathy & $17(1)$ & $6(4)$ & 0 & $/$ \\
hepatic toxicity & $32(21)$ & $8(5)$ & 0 & 0 \\
renal toxicity & $17(11)$ & $3(2)$ & 0 & 0 \\
acneiform rash & 0 & 0 & $1(1)$ & 0 \\
hypermagnesemia & $14(9)$ & $38(25)$ & 0 & 0 \\
allergic reaction to cetuximab & $6(4)$ & 0 & $3(2)$ & 0 \\
allergic reactions to oxaliplatin & $1(1)$ & 0 & 0 & 0 \\
arterial hypertension & $3(2)$ & $9(6)$ & 0 & 0 \\
proteinuria & $20(13)$ & $18(12)$ & 0 & $/$ \\
bleeding & $6(4)$ & 0 & $1(1)$ & 0 \\
thromboembolic events & 0 & $7(5)$ & 0 & 0 \\
wound complications & 0 & 0 & 0 & $/$ \\
perforation & 0 & 0 & 0 & 0 \\
arthralgia & $17(11)$ & $2(2)$ & 0 & 0 \\
bladder infection & $/$ & $3(2)$ & 0 & 0 \\
\hline
\end{tabular}

* 1 patient had febrile neutropenia; / = this grade of adverse effect does not exist; $0=$ this grade of adverse effect was not observed in our study

carcinomatous lymphangiosis, vascular and perinevral invasion, which speak of a greater biological aggressiveness of the disease and are important for decision on adjuvant systemic treatment in stages II and III, have not proved to be predictive factors of a worse outcome of the disease, although the difference in size of the primary T4 tumour was marginal $(p=0.08)$. We hypothesize that the cause of this could be the insufficient number of patients with such histological parameters as well as the short observation period (median observation period of 15 months), since the median OS in these patients has not yet been reached. Another possible cause may be that these histological parameters are not significantly relevant for the further course of the disease. According to the data from the literature, locally advanced primary tumour, with the infiltration of the free surface of serosa, and the incidence of a large number of regional lymph nodes, vascular invasion and carcinomatous lymphangiosis are independent prognostic factors for worse outcome of the operable disease. ${ }^{2,19}$ In the case of disseminating disease this parameters also predicts aggressive course although their importance in the spread of disease is not fully defined.

The percentage of mutations in the codon 12 and 13 of the KRAS gene was found in $42 \%$ of pa- 
tients, which is in accordance with the literature data in which 30 to $40 \%$ mutations are reported..$^{20-23}$ According to the data from the retrospective analyses of the published clinical studies the mutations in codon 12 are more frequent than mutations in codon 13, which is also confirmed by our study. Namely, $73 \%$ of the patients had mutations in codon 12 , and $27 \%$ of the patients had mutations in codon 13. All the mutations analyses in our study were performed on the tumour tissue of the primary tumour. Given the proven high degree of compliance of the mutations between tumour tissue of the primary tumour and metastases, no further analysis of the status of mutations in the KRAS gene in operable metastases or their biopsy has been done. ${ }^{24}$ In our already published retrospective analysis, which included 176 patients, KRAS mutations in the genes were present in $30 \%$ of the patients and more often in codon 12 than in codon 13 (84\% in codon 12 vs. $16 \%$ in codon 13$).{ }^{24}$

The median OS of patients with the non-mutated KRAS gene was shorter in our study than in patients with mutant KRAS genes, but, the difference was not statistically significant $(\mathrm{p}=0.47)$, suggesting that the mutation status in the KRAS gene predicts response to the treatment with EGFR inhibitors, but is not a prognostic factor for survival of the patients. In some patients with a wtKRAS gene the presence of the V600E mutation in the BRAF gene most likely can be the cause of a shorter survival, since the presence of the V600E mutation in the BRAF gene, according to retrospective reports of previous clinical studies, indicates a worse outcome of metastatic disease. ${ }^{25-28}$ The mutation of the V600E in the BRAF gene was present only in patients with a non-mutated KRAS gene, which was expected in accordance with previous reports of mutually exclusive exclusion of the simultaneous presence of the both mutations. ${ }^{24}$ The median OS of patients with mutations in codon 12 was longer than in patients with mutations in codon 13 but was not significant $(\mathrm{p}=0.41)$. According to the data from the literature, patients with mutations in codon 13 have a shorter survival than patients with mutations in codon 12. Patients with mutations in codon 13 respond to treatment with EGFR inhibitors, otherwise in a lower percentage as patients without mutations in the KRAS gene. The retrospective analysis of the efficacy data of these patients with mutations both in codon 12 and codon 13 treated with another EGFR panitumum$\mathrm{ab}$ inhibitor did not confirm this. ${ }^{28-30}$ In standard treatment with regard to systemic treatment recommendations, patients with mutant KRAS gene do not receive a therapy with EGFR inhibitors. ${ }^{15}$ In our study, we also analysed the efficacy of systemic therapy in patients with mutant KRAS gene with respect to the presence of mutations in codons 12 and 13, none of whom received EGFR therapy. In our analysis, however, we found that patients with mutations in codon 13 respond to a higher percentage to systemic therapy than patients with mutations in codon 12 (47\% vs. 23\%). The definitive conclusions of this analysis are difficult to give, given that the patients with mutations in codon 13 are in lower number than patients with mutations in codon 12 (27\% vs. $73 \%)$. These mutations are less frequent in accordance with the already published data from our retrospective study (16\% vs. $84 \%$ ) and other published data (16-28\% mutations in the codon 13 KRAS gene). ${ }^{28-30}$ However, given that they have a shorter survival than patients with mutations in codon 12, but respond in a higher percentage to the first treatment, we believe that results of this analysis will help in the treatment of these patients, and in particular in reducing the disease burden and improving the quality of life.

In our study, we also analysed TTP of the disease in patients with operable disease. There were 64 such patients, with operable primary tumour without metastases, 16 patients with rectal cancer and 48 with colon carcinoma. Median time to recurrence of the disease in patients after primary treatment of operable disease with a non-mutated $K R A S$ gene was shorter than in patients with mutated KRAS gene (20 vs. 21 months), but the difference was not significant $(\mathrm{p}=0.88)$. We assume that the V600E mutation in the BRAF gene, which was found in four patients with a non-mutated KRAS gene contributes for such results. With this, we confirmed the prognostic importance of the V600E mutation in the BRAF gene for early progression and worse outbreaks in patients with a non-mutated KRAS gene. ${ }^{31,32}$ The TTP of the disease in patients primarily treated for operable disease with operation was longer in those who had mutations in the KRAS codon 12 than in those with mutations in codon 13 (21 vs. 16 months), otherwise the difference was not significant $(p=0.53)$.

Regarding the presence of the V600E mutation in the BRAF gene in our study, as previously stated it was found in four patients, representing 3\% of all patients. According to the literature, the frequency of this mutation is from 5 to $10 \% .^{2,3,9,13}$ Also, according to our previous retrospective analysis, the V600E mutation in the BRAF gene was present at a higher percentage of patients, in $7.4 \%{ }^{24}$ It is assumed that the specificity of the patients involved 
with such primary tumour characteristics may be due to the fact that the V600E mutation was present in a lower percentage. All four patients with the V600E mutation had colon cancer, three patients were previously on adjuvant treatment, and one had a primary disseminated disease. In a retrospective analysis of the study involving 94 patients with rectal carcinoma, none of the V600E mutations in the BRAF gene were detected in either patient. ${ }^{33}$ This suggests that this mutation is probably less important in the development of rectal cancer. The time before progression of the disease in patients after primary treatment is longer for those who did not have the V600E mutation in the BRAF gene than in the V600E mutation (20 to 12 months), but the difference was not significant $(\mathrm{p}=0.14)$. The cause is most likely a small number of patients with this mutation, but such result suggests a worse prognosis of these patients and an earlier recurrence of the disease. In the survival analysis, patients without V600E mutations had a significantly longer median OS than those with V600E mutation (59.2 vs. 27.6 months; $\mathrm{p}=0.05$ ). In a retrospective analysis of 176 patients, 13 patients with the V600E mutation in the BRAF gene had a significantly lower expected median survival than patients without this mutation (44.9 vs. 107.4 months; $p=0.04 .{ }^{24}$ Both studies, both the previous retrospective and the current prospective, confirm the prognostic importance of the V600E mutation in the $B R A F$ gene for predicting a worse disease outcome with shorter patient survival.

Furthermore, we examined the response rate according to the type of systemic treatment in patients with non-mutated KRAS gene. There were 88 such patients. Three hundred and sixty patients with a non-mutated KRAS gene received the first line of systemic treatment with EGFR-inhibitor cetuximab in combination with oxaliplatin or irinotecan and fluoropyrimidines, 35 patients with a non-mutated KRAS gene received the first line of systemic treatment with angiogenesis inhibitor bevacizumab in combination with oxaliplatin or irinotecan and fluoropyrimidines. A higher percentage of objective responses (both complete and partial), both for treatment and stability of the disease was achieved in patients with non-mutated $K R A S$ gene receiving cetuximab in combination with oxaliplatin or irinotecan and fluoropyrimidines.

The definitive conclusions about this analysis cannot be made within our research, since this is a small number of patients, and the number of patients was not balanced in number. In addition, the types of systemic chemotherapy that patients received differ. However, it was found that patients with a non-mutated KRAS gene had a better response to systemic first-line treatment involving cetuximab regardless of the type of systemic chemotherapy as patients with the non-mutated KRAS gene that received angiogenesis inhibitor bevacizumab in combination with systemic chemotherapy.

Colorectal adenocarcinoma is a heterogeneous disease. We do not know all the factors that influence the course of the disease and the response to specific systemic therapy. The determination of other mutations in the KRAS gene, such as mutations in codon 61 and 146, and mutations in the NRAS gene, has been standard in clinical practice since 2014. The frequency of mutations is about $15 \%$ and the patients with a non-mutated RAS gene have about $40 \%$ of this mutation. In our retrospective analysis, we found $17 \%$ of these additional mutations, which is consistent with reports from previous literature and they are predictive for response to EGFR inhibitor therapy. Patients treated with first-line anti-EGFR targeted therapy without these additional mutations in RAS gene, had longer survival and time to progression compared to the patients who had them. Our results confirmed predictive value of these mutations in RAS gene for anti-EGFR targeted therapy. However, our group of patients is moderate, but it is consisted with previously published data. ${ }^{33,34}$

Adverse effects of systemic treatment were evaluated according to NCI-CTC, version 4.03. They were mostly of Grade 1 and 2. There were $2 \%$ of G3 diarrhea and hypermagnesemia and 1\% of G3 bleeding. One patient had G4 adverse effect (febrile neutropenia). In our study, adverse effects were less common than in previous published literature. ${ }^{35-47}$ None of the patients died of adverse effects.

However, all patients with RAS non-mutated genes do not respond to treatment with anti-EGFR inhibitors. Other biomarkers have to be determined. One of them is mismatch repair- deficiency (MSI-H), which is present in 3.5\% to $5.0 \%$ of mCRC. ${ }^{48,49}$ Immunotherapy with checkpoint inhibitors is recommended in second-line therapy in these patients. In clinical trials, there are other biomarkers in response to specific systemic therapy, such as amfiregulin and epiregulin, PI3K and PTEN mutations, but are still not recommended in regular clinical practice..$^{50} \mathrm{In}$ order to made definite reasoning on this issue we need more information and more studies, both, retrospective and prospective should be performed. 


\section{Conclusions}

According to the results of our prospective study G3 of tumour differentiation (poorly differentiated adenocarcinoma) and N2 status of regional lymph nodes (more than 3 metastatic regional lymph nodes) show a greater biological aggressiveness of the disease and shorter survival after the first relapse, but do not affect the efficacy of the first-line systemic therapy.

The mutations in the KRAS gene have predictive value for the response to treatment with EGFR inhibitors, but do not have prognostic significance for the survival of patients with disseminated colorectal adenocarcinoma. The KRAS mutations in codon 12 and 13 differently affect the survival of these patients and the response rate of first-line systemic therapy.

The V600E mutation in the BRAF gene does not have the prognostic significance for the decision on the type of first-line systemic therapy, but it predicts a poor prognosis of the disease and shorter survival of these patients at first relapse.

According to our restrospective analysis of additional mutations in NRAS gene and in KRAS codon 61 and 146 are predictive for treatment with anti-EGFR targeted therapy.

\section{Acknowledgement}

The research was founded by Slovenian Research Ageny (ARRS), grant number P3-0321.

\section{References}

1. Cancer in Slovenia 2015. Ljubljana: Institute of Oncology Ljubljana Epidemiology and Cancer Registry, Cancer Registry of republic of Slovenia; 2018.

2. Schmoll1 HJ, Van Cutsem E, Stein A, Valentini V, Glimelius B, Haustermans $\mathrm{K}$, et al. ESMO consensus guidelines for management of patients with colon and rectal cancer. A personalized approach to clinical decision making. Ann Oncol 2012; 23: 2479-516. doi: $10.1093 /$ annonc/mds236

3. Van Cutsem 1 E, Cervantes A, Adam R, Sobrero A, Van Krieken JH, Aderka $D$, et al ESMO consensus guidelines for the management of patients with metastatic colorectal cancer. Ann Oncol 2016; 1386-422. doi: 10.1093/ annonc/mdw235

4. National Comprehensive Cancer Network. NCCN clinical practice guidelines in oncology: colon cancer. V.2.2018. [cited 2018 Jun 15]. Available at: http:// www.nccn.org/profesionals/phisician gls/PDF/colon.pdf

5. National Comprehensive Cancer Network: NCCN clinical practice guidelines in oncology: rectal cancer. V.2.2018 [cited 2018 Aug 3]. Available at: http:// www.nccn.org/profesionals/phisician gls/PDF/colon.pdf

6. Biagi JJ, Raphael MJ, Mackillop WJ, Kong W, King WD, Booth CM. Association between time to initiation of adjuvant chemotherapy and survival in colorectal cancer. A systemic review and meta-analysis. JAMA 2011; 305: 2335 42. doi: 10.1001/jama.2011.749
7. Ocvirk J. Advances in the treatment of metastatic colorectal carcinoma. Radiol Oncol 2009; 43: 1-8. doi: 10.2478/v10019-009-0004-1

8. Meyerhardt JA, Mayer RJ. Systemic therapy for colorectal cancer. N Engl Med 2005; 352: 476-87. doi: 10.1053/j.gastro.2008.02.098

9. Esin E, Yalcin S. Maintenance strategy in metastatic colorectal cancer: a systematic review. Cancer Treat Rev 2016; 42: 82-90. doi: 10.1016/j. ctrv.2015.10.012

10. Advanced colorectal cancer: ESMO clinical practice guidelines for diagnosis, treatment. Ann Oncol 2010; 21: v93- 7. doi: 10.1093/annonc/mdq222

11. Markowitz SD, Bertagnolli MM. Molecular basis of colorectal cancer. $N$ Eng J Med 2009; 361: 2449-60. doi: 10.1056/NEJMra0804588

12. De Roock W, Jonker DJ, Di Nicolantonio F, Sartore-Bianchi A, Tu D, Siena $S$, Lamba S, et al. Association of KRAS p.G13D mutation with outcome in patients with chemotherapy-refractory metastatic colorectal cancer treated with cetuximab. JAMA 2010; 304: 1812- 20. doi: 10.1001/jama.2010.1535

13. De Roock W, Claes B, Bernasconi D, De Schutter J, Biesmans B, Fountzilas $\mathrm{G}$, et al. Effect of KRAS, BRAF, NRAS, and PIK3CA mutations on the efficacy of cetuximab plus chemotherapy in chemotherapy-refractory metastatic colorectal cancer: a retrospective consortium analysis. Lancet Oncol 2010; 11: 753-62. doi: 10.1016/S1470-2045(10)70130-3

14. Tol J, Nagtegaal ID, Punt CJA. BRAF mutation in metastatic colorectal cancer. N Engl J Med 2009; 361: 98-99. doi: 10.3978/j.issn.2078-6891.2015.077

15. Samowitz WS, Sweeney C, Herrick J, Albertsen H, Levin TR, Murtaugh MA, et al. Poor survival associated with the BRAF V600E mutation in microsatellite- stable colon cancer. Caner Res 2005; 65: 6063-9. doi: 10.1158/00085472.CAN-05-0404

16. Ličar A, Cerkovnik P, Novaković S. Distribution of some activating KRAS and BRAF mutations in Slovene patients with colorectal cancer. Med Oncol 2011; 28: 1048-53. doi: 10.1007/s12032-010-9631-z

17. Fariña-Sarasqueta $A$, van Lijnschoten $G$, Moerland $E$, Creemers GJ, Lemmens VE, Rutten HJ, et al. The BRAF V600E mutation is an independent prognostic factor for survival in stage II and stage III colon cancer patients. Ann Oncol 2010; 21: 2396-402. doi: 10.1093/annonc/mdq258

18. Eisenhauer EA, Therasse P, Bogaerts J, Schwartz LH, Sargent D, Ford R, et al. New response evaluation criteria in solid tumors: revised RECIST guideline (version 1.1). Eur J Cancer 2009; 45: 228-47. doi: 10.1016/j.ejca.2008.10.026

19. Marzouk O, Schofield J. Review of histopathological and molecular prognostic features in colorectal cancer. Cancers 2011; 3: 276-81. doi: 10.3390/ cancers 3022767

20. Ličar A, Cerkovnik P, Ocvirk J, Novaković S. KRAS mutations in Slovene patients with colorectal cancer: frequency, distribution and correlation with the response to treatment. Int J Oncol 2010; 36: 1137-44. doi: 10.3892/ ijo_00000596.

21. Artale S, Sartore- Bianchi A, Veronese SM, Gambi V, Sarnataro CS, Gambacorta M, et al. Mutations of KRAS and BRAF in primary and matched metastatic sites of colorectal cancer. J Clin Oncol 2008; 26: 4217- 9. doi: 10.1200/JCO.2008.18.7266

22. Bokemeyer C, Van Cutsem E, Rougier P, Ciardiello F, Heeger S, Schlichting $M$, et al. Addition of cetuximab to chemotherapy as first-line treatment for KRAS wild-type metastatic colorectal cancer: pooled analysis of the CRYSTAL and OPUS randomised clinical trials. Eur J Cancer 2012; 48: 146675. doi: 10.1016/j.ejca.2012.02.057

23. Artale S, Sartore- Bianchi A, Veronese SM, Gambi V, Sarnataro CS, Gambacorta M, et al. Mutations of KRAS and BRAF in primary and matched metastatic sites of colorectal cancer. J Clin Oncol 2008; 26: 4217-9. doi: 10.1200/JCO.2008.18.7286

24. Rebersek M, Boc M, Škerl P, Benedik J, Hlebanja Z, Volk N, et al. Efficacy of first- line systemic treatment in correlation with BRAF V600E and different KRAS mutations colorectal cancer - a single institution retrospective analysis. Radiol Oncol 2011; 45: 285-91. doi: 10.2478/v10019-011-0039-y

25. Lambrechts D, De Roock W, Prenen H, De Schutter J, Jacobs B, Biesmans B, et al. The role of KRAS, BRAF, NRAS and PIK3CA mutations as a markers of resistance of cetuximab in chemorefractory metastatic colorectal cancer. $J$ Clin Oncol 2009; 27: 9020. doi: 10.1158/1078-0432.CCR-08-2961

26. Tejpar S, De Roock W. PIK3CA, BRAF and KRAS mutations and outcome prediction in chemorefractory metastatic colorectal cancer (mCRC) patients treated With EGFR targeting monoclonal antibodies (MoAbs): results of a European Consortium. [abstract]. Eur J Cancer Supp/ 2009; 7: 322. No. 6005 
27. Loupakis F, Ruzzo A, Cremolini C, Vincenzi B, Salvatore L, Santini D, et al. KRAS codon 61,146 and BRAF mutations predict resistence to cetuximab plus irinotecan in KRAS codon 12 and 13 wild-type metastatic colorectal cancer. Br J Cancer 2009; 101: 715-21. doi: 10.18632/oncotarget.13697

28. Tejpar S, Celik I, Schlichting M, Sartorius U, Bokemeyer C, Van Cutsem E. Association of KRAS G13D tumor mutations with outcome in patients with metastatic colorectal cancer treated with first-line chemotherapy with or without cetuximab. J Clin Oncol 2012; 30: 3570-7. doi: 10.1200/ JCO.2012.42.2592

29. Peeters M, Douillard JY, Van Cutsem E, Siena S, Zhang K, Williams R, et al. Mutant KRAS codon 12 and 13 alleles in patients with metastatic colorectal cancer: assessment as prognostic and predictive biomarkers of response to panitumumab. J Clin Oncol 2013; 31: 759-65. doi: 10.1200/ JCO.2012.45.1492. Epub 2012 Nov 26.

30. Roth AD, Tejpar S, Dolorenzi M, Yan P, Fiocca R, Klingbiel D, et al. Prognostic role of KRAS and BRAF in stage II and III resected colon cancer: results of the translational study on the PETACC-3, EORTC 40993, SAKK 60-00 trial. J Clin Oncol 2010: 28: 466-74. doi: 10.1200/JCO.2009.23.3452.

31. Fujiyoshi K, Yamamoto G, Takahashi A, Arai Y, Yamada M, Kakuta M, et al. High concordance rate of KRAS/BRAF mutations and MSI-H between primary colorectal cancer and corresponding metastases. Oncol Rep 2017; 37: 785-92. doi: 10.3892/or.2016.5323.

32. Gaedcke J, Grade M, Jung K, Schirmer M, Jo P, Obermeyer C, et al. KRAS and BRAF mutations in patients with rectal cancer treated with preoperative chemoradiotherapy. Radiother Oncol 2010; 94: 76-81. doi: 10.1016/j. radonc.2009.10.001

33. Pietrantonio F, Cremolini C, Petrelli F, Di Bartolomeo M, Loupakis F, Maggi $C$, et al. First-line anti-EGFR monoclonal antibodies in panRAS wild-type metastatic colorectal cancer: a systematic review and meta-analysis. Crit Rev Oncol Hematol 2015; 96: 156-66. doi: 10.1016/j.critrevonc.2015.05.016

34. Sorich MJ, Wiese MD, Rowland A, Kichenadasse G, McKinnon RA, Karapetis CS, et al. Extended RAS mutations and anti-EGFR monoclonal antibody survival benefit in metastatic colorectal cancer: a meta-analysis of randomized, controlled trials. Ann Oncol 2015; 26: 13-21. doi: 10.1093/annonc/mdu378

35. Scartozzi M, Galizia E, Chiorrini S, Giampieri G, Berardi R, Pieratoni C, et al. Arterial hypertension correlates with clinical outcome in colorectal cancer treated with first- line bevacizumab. Clin Oncol 2009; 20: 227-30. doi: 10.1093/annonc/mdn637

36. Mir O, Ropert S, Alexandre J, Goldwasser F. Hypertension as a surrogate marker for the activity of anti- VEGF agents. Ann Oncol 2009; 20: 967-70. doi: 10.1093/annonc/mdp206

37. Poole C, Gardiner J, Twelves C, Johnston P, Harper P, Cassidy J, et al. Effect of renal impairment on the pharmacokinetics and tolarebility of capecitabine (Xeloda) in cancer patients. Cancer Chemother Pharmacol 2002; 49: 225-34. doi: 10.1007/s00280-001-4808-0

38. Cassidy J, Twelves C, Van Cutsem E, Hoff P, Bajetta E, Boyer M, et al. First-line oral capecitabine therapy in metastatic colorectal cancer: a favorable safety profile compared with intravenous 5-fluorouracil/leucovorin. Ann Oncol 2002; 13: 566-75. doi: 10.1200/JCO.2001.19.21.4097

39. Hoskins JM, Goldberg RM, Qu P, Ibrahim JG, McLeod HL. UGT1A1* 28 genotype and irinotecan- induced neutropenia: dose matters. J Nati Cancer Inst 2007; 99: 1290-421. doi: 10.1200/JCO.2009.21.7943

40. Hoskins JM, McLeod HL. UGT1A and irinotecan toxicity: keeping it in the family. J Clin Oncol 2009; 27: 2419-21. doi: 10.2217/pgs.10.95

41. Pereg D, Lishner M. Bevacizumab treatment for cancer treatment with cardiovascular disease: a double edged sword? Eur Heart J 2008; 29: 2325-26. doi: 10.1093/eurheartj/ehn384.

42. Scappaticci FA, Skillings JR, Holden SN, Gerber HP, Miller K, Kabbinavar F, et al. Arterial thromboembolic events in patients with metastatic colorectal cancer treated with chemotherapy and bevacizumab. J Nati Cancer Inst 2007; 1232-9. doi: 0.1093/jnci/djm086

43. Lee CS, Ryan EJ, Glen A Doherty GA. Gastro-intestinal toxicity of chemotherapeutics in colorectal cancer: the role of inflammation. World $J$ Gastroenterol 2014; 20: 3751-61. doi: 10.3748/wjg.v20.i14.3751

44. Ocvirk J, Rebersek M. Management of cutaneous side effect of cetuximab therapy with K1 creme. Radiol Oncol 2008; 42: 215-24. doi: 10.2478/ v10019-008-0022-4
45. Ocvirk J, Cencelj S. Management of cutaneous side-effect of cetuximab therapy in patients with metastatic colorectal cancer. JEADV 2010; 24: 4539. doi: 10.1111/j.1468-3083.2009.03446.x

46. Ocvirk J, Rebersek M, Boc M, Mesti T, Ebert Moltara M. Prophylactic use of $\mathrm{K} 1 \mathrm{crem}$ for reducing skin toxicity during cetuximab treatment in patients with metastatic colorectal cancer (mCRC). J Clin Oncol 2016; 28(15 Suppl): V124. doi: 10.1200/jco.2010.28.15_suppl.e14011.

47. Siena S, Glynne-Jones R, Adenis A, Thaler J, Preusser P, Aguilar EA, et al. Reduced incidence of infusion- related reactions in metastatic colorectal cancer during treatment with cetuximab plus irinotecan with combined corticosteroid and antihistamin premedication. Cancer 2010, 116: 1827-37. doi: 10.1002/cncr.24945

48. Venderbosch, ID Nagtegaal, TS Maughan, CG Smith, JP Cheadle, D Fisher, et al. Mismatch repair status and BRAF mutation status in metastatic colorectal cancer patients: a pooled analysis of the CAIRO, CAIRO2, COIN, and FOCUS studies. Clin Cancer Res 2014 20: 5322-30. doi: 10.1158/1078-0432. CCR-14-0332

49. Le DT, Uram JN, Wang H, Bartlett BR, Kemberling H, Eyring AD, et al. PD-1 blockade in tumors with mismatch-repair deficiency. N Engl J Med 2015; 372: 2509-20. doi: 10.1056/NEJMoa1500596

50. Therkildsen C, Bergmann TK, Henrichsen-Schnack T, Ladelund S, Nilbert $M$. The predictive value of KRAS, NRAS, BRAF, PIK3CA and PTEN for antiEGFR treatment in metastatic colorectal cancer: a systematic review and meta-analysis. Acta Oncol 2014; 53: 852-64. doi: 10.3109/0284186X.201 4.895036 